\title{
Transcultural adaptation into Portuguese of an instrument for pain evaluation based on the biopsychosocial model
}

\author{
Adaptação transcultural para a língua portuguesa de um \\ instrumento de orientação para avaliação da dor
}

\author{
Monique Rocha Peixoto dos Santos ${ }^{[a]}$, Leandro Calazans Nogueira ${ }^{[a, b]}$, Ney Armando Meziat-Filho ${ }^{[a, b]}$, \\ Rob Oostendorp ${ }^{[\mathrm{c}, \mathrm{d}]}$, Felipe José Jandre dos Reis ${ }^{[\mathrm{a}, \mathrm{e}]^{*}}$ \\ [a] Instituto Federal de Educação, Ciência e Tecnologia do Rio de Janeiro (IFRJ), Rio de Janeiro, RJ, Brazil \\ [b] Centro Universitário Augusto Motta (UNISUAM), Rio de Janeiro, RJ, Brazil \\ [c] Radboud University Medical Centre, Nijmegen, Netherlands \\ [d] Vrije Universiteit Brussel, Brussels, Belgium \\ [e] Universidade Federal do Rio de Janeiro (UFRJ), Rio de Janeiro, RJ, Brazil
}

\section{Abstract}

Introduction: Pain is an individual experience influenced by multiple interacting factors. The "biopsychosocial" care model has gained popularity in response to growing research evidence indicating the influence of biological, psychological, and social factors on the pain experience. The implementation of this model is a challenge in the practice of the health professional. Objective: To perform the transcultural adaptation of the SCEBS method into Brazilian Portuguese. Methods: The instrument was translated and applied to 50 healthy subjects and 50 participants with non-specific chronic pain in the spine. The process of cross-cultural adaptation included the following steps: transcultural adaptation, content analysis of the scale, pre-test, revision, back-translation review, cross-cultural adaptation, revised text correction and final report. Results: The translated and adapted 51-item Portuguese version of the SCEBS method produced an instrument called SCEBS-BR. In the assessment by the target

\footnotetext{
MRPS: BS, e-mail: monique.julio@hotmail.com LCN: PhD, e-mail: leandro.nogueira@ifrj.edu.br NAMF: PhD, e-mail: neymeziat@gmail.com R0: PhD, e-mail: ostendorp.rob@gmail.com FJJR: PhD, e-mail: felipe.reis@ifrj.edu.br
} 
population, 50 adult users of the Brazilian Unified Health System answered the questionnaire and showed good understanding of the instrument on the verbal rating scale. Conclusion: The SCEBS-BR was proved to be easily understandable, showing good semantic validation regardless of schooling level or age, and can be considered adequate for clinical use.

Keywords: Pain. Chronic Pain. Cross-Cultural Comparison. Lower Back Pain. Neck Pain.

\section{Resumo}

Introdução: A dor é uma experiência individual influenciada por aspectos multifatoriais. O modelo de cuidado "biopsicossocial" adquiriu popularidade em resposta à crescente evidência de pesquisas indicando a influência de fatores biológicos, psicológicos e sociais na experiência de dor. A implantação deste modelo é um desafio na prática do profissional de saúde. Objetivo: Realizar a adaptação transcultural do Método SCEBS para o português do Brasil. Métodos: 0 instrumento foi traduzido e aplicado a 50 indivíduos saudáveis e 50 pacientes com dor crônica não específica na coluna. 0 processo de adaptação transcultural incluiu as seguintes etapas: adaptação transcultural, análise de conteúdo da escala, pré-teste, revisão, revisão de back-translation, adaptação transcultural, correção de texto revisada e relatório final. Resultados: A versão portuguesa traduzida e adaptada de 51 itens do método SCEBS produziu um instrumento denominado SCEBS-BR. Na avaliação da população-alvo, 50 usuários adultos do Sistema Único de Saúde responderam ao questionário e mostraram boa compreensão do instrumento na escala de classificação verbal. Conclusão: O SCEBS-BR demonstrou ser facilmente compreensível, mostrando boa validação semântica independentemente do nível de escolaridade ou idade e considerado adequado para o uso clínico.

Palavras-chave: Dor. Dor Crônica. Comparação Transcultural. Dor Lombar. Dor Cervical.

\section{Introduction}

The International Association for the Study of Pain (IASP) defines pain as "an unpleasant sensory and emotional experience associated with actual or potential tissue damage, or described in terms of such damage" [1]. Recently, Williams and Craig [2] suggested an update to this definition: "a distressing experience associated with actual or potential tissue damage with sensory, emotional, cognitive and social components". In both definitions, pain is an experience that involves different domains that go beyond the simple response to a stimulus.

Despite the importance of recognizing pain as a multifactorial construct, it is still common for health professionals to direct their efforts towards the investigation and treatment of biological factors in pain management. The fragmentation of biological, psychological, social, and environmental factors contributes to difficulties in understanding the broader contexts of pain and its management $[1,3,4]$. Currently, the biopsychosocial model (BPS) aims to integrate these multiple factors and their contributions to health conditions.

Since the introduction of the BPS model, there has been a considerable shift towards the use of this model for the diagnosis and treatment of musculoskeletal disorders such as lower back and neck pain [5, 6]. Indeed, the biomedical model, focused on anatomical structures and biomechanics, is not enough to explain many symptoms of pain conditions [7,8]. In the biopsychosocial perspective, biological variables, psychological condition, and social setting are dynamically related to pain, disability, and emotional disorders [9 - 11]. Consequently, pain can be seen as the result of the influence of biological, cognitive, and emotional factors, and the environment [5].

Despite recognizing in pain, the interaction of several factors, health professionals may experience difficulties in addressing all necessary aspects. The SCEBS method (Somatic, Cognition, Emotion, Behavior and Social) is an instrument that is easy to apply in clinical practice that presents questions to address the patient's experience within the BPS model. 
The main purpose of the SCEBS method is to be a guide for clinical interview based on the BPS model that contains questions comprising the three dimensions of pain: Somatic or biological, psychological (Cognition, Emotion, Behavior) and Social [12]. In Brazil, there is currently no instrument to guide clinicians to consider all aspects of the BPS model in the assessment of patients with lower back or neck pain. Therefore, the objective of the present study was to perform the cross-cultural adaptation of the SCEBS method into Portuguese. We believe that the availability of this instrument as an evaluation guide can help to facilitate a patient-centered approach, therapistpatient communication, and adherence to treatment by people with lower back and neck pain.

\section{Methods}

Study design

This study is characterized as a transcultural adaptation. All the ethical aspects of the CNS / MS 466/2012 resolution were respected, and the project was approved by the Ethics and Research Committee of the Instituto Federal do Rio de Janeiro (IFRJ) and registered (CAEE 59001316.9.0000.5268). All participants signed the informed consent form and were informed about the nature of the study and the protocol.

\section{Procedures}

The process of cross-cultural adaptation included the following steps: preparation, translation, reconciliation (synthesis), back-translation, backtranslation review, harmonization (comparison of backward versions), cross-cultural adaptation (content analysis; pre-test, revised text) and final version, respecting the methodology recommended for this study design [13 - 15].

The original SCEBS method was translated from English into Portuguese by two native English translators, fluent in Portuguese and experienced in translation ( $\mathrm{T} 1$ and $\mathrm{T} 2$ ). In the reconciliation stage (synthesis), the researchers analyzed the T1 and T2 versions together with the original questionnaire, giving rise to the T12 version.
In the back-translation step, two independent backtranslators (RT1 and RT2) participated, one having English as a mother tongue and fluency in Portuguese and the other having Brazilian Portuguese as a mother tongue and fluency in English, neither of whom participated in the translation step. This step was performed to produce the RT12 version. All versions (original, T1, T2, T12, RT1, RT2, RT12) were reviewed by the Translation Group and the Committee of Experts. The Committee of Experts followed the guidelines of Beaton et al. [14], being oriented to carry out the careful reading of the instrument and its versions to propose changes and corrections, evidencing the reasons. The goal of this step was to obtain clear and accessible language. Semantic equivalence principles were considered, such as the referential meaning of the words used and the general meaning of the questions, as well as the response options or orientation of the instrument originating from the translation.

The pre-test of the pre-final version of the SCEBS method had the objective of eliminating any item not comprehended by more than $20 \%$ of the sample, following the recommendations of Nusbaum et al. [16].

At this moment a pre-test version of the instrument was applied to asymptomatic and symptomatic individuals. The asymptomatic group comprised people of both genders, with no age limit, and without chronic musculoskeletal pain. The symptomatic group comprised volunteers of both genders, aged over 18 years, with acute, subacute or chronic pain classified as non-specific pain (without defined clinical diagnosis), located in the lumbar or neck regions, who were in treatment in the physiotherapy service of the IFRJ-Campus, Realengo. Participants with significant cognitive impairment were not considered eligible for the study due to the possibility of this compromising their understanding of the instructions. In the case of individuals with reading difficulties, the evaluator read out the instructions, questions, and options. In both groups the selection was performed for convenience.

The guiding question for this early stage was, "Did you understand the sentence?" The participant should indicate a value on a 5-point Likert scale with a minimum value of "0" ("I understood just a little") and a maximum value of "4" ("I completely understood"). It was established that the first three values $(0,1$, and 2) would be considered indicators of insufficient 
understanding and, in this case, the sentence should be reviewed by the research team [17]. In the sixth step of the study, all reports were submitted to the Expert Committee and evaluated, and a final version was sent to the original author for approval. The final version of the instrument was reviewed by a specialist in Portuguese.

\section{Data analysis methodology}

Data were tabulated in a spreadsheet and analyzed using SPSS 20 software for Windows. Data from variables gender, schooling, degree of comprehension of the sentences were presented as absolute and relative frequency measures. Age was presented as mean and standard deviation.

\section{Results}

Phases of transcultural translation and adaptation

The instrument was translated and adapted by a group of translators, back translators and specialists (three physiotherapists, an occupational therapist, two psychologists, and a physician). All specialists have more than five years of experience in the management of people with musculoskeletal pain.

During the back-translation phase, adaptations were made to make the translation of the SCEBS method as simple and comprehensible as possible. In this sense, the expression queixas was replaced by sintomas in most of the items, in order to differentiate the symptom itself from the act of complaining. In the somatic dimension, the sentence Quais são a natureza in item 3 was modified to Quais são os tipo, in order to facilitate understanding of the question. In item 5 the definite article os was replaced by the demonstrative pronoun esses, to represent the symptoms mentioned by the individual himself. In item 7 the word mover was changed to movimentar, and the word rigidez in item 8 was changed to dificuldade para se movimentar because the word rigidez alone is not clear enough.

Regarding the psychological dimension, on the cognition axis the word própria was removed from item 13 to avoid redundancies, and dos seus sintomas was added at the end of the sentence. To make the meaning of item 14 clearer, the pronoun você was inserted at the beginning of the sentence. In item 17 the phrase nesses momentos was replaced by nos momentos que tem os sintomas. In the subtitle Ideias sobre influência pessoal sobre as queixas, the phrase sobre as queixas was replaced by nas queixas. In item 19, in order to make the question clearer, the phrase Você pessoalmente tem has been replaced by Você acha que tem. In item 20 the phrase você é capaz was modified to você acha que é capaz. Item 21 was modified from Se é, como to Se sim, de que maneira? for clarity. And in item 23 the sentence os problemas são resolvidos has been replaced by os seus sintomas melhoram since the question is related to persistent pain.

Regarding the emotion axis of the psychological dimension, in item 25 the end of the sentence como você se sente was extended to como você se sente em relação a eles, and in item 26 the phrase as queixas perturbam was changed to você acha que seus sintomas afetam. In items 27,28 , and 29 the question você está was replaced by você se sente (in item 27) and by você se considera (in items 28 and 29) so as not to induce the answer.

On the behavioral axis of the psychological dimension, in item 31 the condition se tem was modified to quando tem because the original sentence could imply that the individual has or does not have the symptoms, which was not the purpose of the question. In item 35, to the question Até que ponto? was added os sintomas prejudicam suas atividades, with the intention of making the meaning clearer and less dependent on the previous question. In item 36 the verb tense deixou was changed to deixa, with the purpose of keeping a consistent verb tense in every question. In item 38 the word sobre was replaced by em relação $a$ in order to adjust the sentence.

The verb notam has been replaced by percebem in items 39 (from the psychological dimension, in the behavior axis), 42 and 43 (from the social dimension) to leave the questions clearer. The subtitle Conversando sobre os problemas has been changed to Falando sobre as queixas since the individual will be reporting their complaints.

Finally, in the social dimension, item 47, Seu parceiro tem ideia do motivo das suas queixas, has been changed as follows: Para o seu parceiro, de onde é que surgem os seus sintomas? This was to facilitate understanding and reduce the range of possible responses. Table 1 illustrates the transcultural adaptation of the SCEBS method. 
Table 1 - The transcultural adaptation of the SCEBS method

\begin{tabular}{|c|c|c|c|c|}
\hline $\mathrm{N}^{\circ}$ & Original & $\mathrm{T} 12$ & RT12 & Final Vesion \\
\hline A.1. & S = Somatic Dimension & $S=$ Dimensão Somática & $S=$ Somatic Dimension & $S=$ Dimensão Somática \\
\hline 1 & What are your complaints? & Quais são as suas queixas? & What are your complaints? & Quais são as suas queixas? \\
\hline 2 & $\begin{array}{l}\text { When did the complaints } \\
\text { begin? }\end{array}$ & $\begin{array}{c}\text { Quando as suas queixas } \\
\text { começaram? }\end{array}$ & $\begin{array}{c}\text { When did your complaints } \\
\text { begin? }\end{array}$ & $\begin{array}{c}\text { Quando os seus sintomas } \\
\text { começaram? }\end{array}$ \\
\hline 3 & $\begin{array}{l}\text { What are the nature, the } \\
\text { location, and the intensity of } \\
\text { the complaints? }\end{array}$ & $\begin{array}{c}\text { Quais são a natureza, a } \\
\text { localização e a intensidade } \\
\text { das suas queixas? }\end{array}$ & $\begin{array}{c}\text { What are the nature, the } \\
\text { location and the intensity of } \\
\text { your complaints? }\end{array}$ & $\begin{array}{c}\text { Quais são o tipo, a } \\
\text { localização e a intensidade } \\
\text { dos seus sintomas? }\end{array}$ \\
\hline 4 & $\begin{array}{l}\text { How often do the symptoms } \\
\text { occur? }\end{array}$ & $\begin{array}{l}\text { Com que frequência os } \\
\text { sintomas ocorrem? }\end{array}$ & $\begin{array}{l}\text { How often do the symptoms } \\
\text { occur? }\end{array}$ & $\begin{array}{l}\text { Com que frequência os } \\
\text { sintomas ocorrem? }\end{array}$ \\
\hline 5 & $\begin{array}{l}\text { How long do the symptoms } \\
\text { last? }\end{array}$ & $\begin{array}{l}\text { Quanto tempo duram os } \\
\text { sintomas? }\end{array}$ & $\begin{array}{l}\text { How long do your symptoms } \\
\text { last? }\end{array}$ & $\begin{array}{c}\text { Quanto tempo duram esses } \\
\text { sintomas? }\end{array}$ \\
\hline 6 & $\begin{array}{l}\text { Have you had these } \\
\text { symptoms before? }\end{array}$ & $\begin{array}{c}\text { Você já teve esses sintomas } \\
\text { antes? }\end{array}$ & $\begin{array}{l}\text { Have you ever had these } \\
\text { symptoms before? }\end{array}$ & $\begin{array}{c}\text { Você já teve esses sintomas } \\
\text { antes? }\end{array}$ \\
\hline 7 & $\begin{array}{c}\text { Can you move your back/ } \\
\text { neck? }\end{array}$ & $\begin{array}{l}\text { Você consegue mover suas } \\
\text { costas ou pescoço? }\end{array}$ & $\begin{array}{c}\text { Can you move your back or } \\
\text { neck? }\end{array}$ & $\begin{array}{l}\text { Você consegue movimentar } \\
\text { suas costas ou pescoço? }\end{array}$ \\
\hline 8 & $\begin{array}{c}\text { Have you experienced any } \\
\text { stiffness? }\end{array}$ & Você teve alguma rigidez? & Have you had any stiffness? & $\begin{array}{l}\text { Você teve alguma dificuldade } \\
\text { para se movimentar? }\end{array}$ \\
\hline 9 & $\begin{array}{c}\text { What do the X-ray results } \\
\text { show? }\end{array}$ & $\begin{array}{c}\text { O que mostram os resultados } \\
\text { do raio- } X \text { ? }\end{array}$ & $\begin{array}{c}\text { What do the x-ray results } \\
\text { show? }\end{array}$ & $\begin{array}{c}0 \text { que mostram os resultados } \\
\text { do raio-X? }\end{array}$ \\
\hline 10 & $\begin{array}{c}\text { What do laboratory tests } \\
\text { show? }\end{array}$ & $\begin{array}{c}0 \text { que mostram os testes de } \\
\text { laboratório? }\end{array}$ & $\begin{array}{c}\text { What do the laboratory tests } \\
\text { show? }\end{array}$ & $\begin{array}{c}0 \text { que mostram os testes de } \\
\text { laboratório? }\end{array}$ \\
\hline A.2. & Psychological Dimension & Dimensão psicológica & Psychological Dimension & Dimensão psicológica \\
\hline A.2.1. & $\begin{array}{l}\mathbf{C}=\text { Cognition } \\
\text { Expectations }\end{array}$ & $\begin{array}{c}\boldsymbol{C}=\text { Cognição } \\
\text { Expectativas }\end{array}$ & $\begin{array}{l}\mathbf{C}=\text { Cognition } \\
\text { Expectations }\end{array}$ & $\begin{array}{l}\boldsymbol{C}=\text { Cognição } \\
\text { Expectativas }\end{array}$ \\
\hline 11 & What do you expect from me? & 0 que você espera de mim? & What do you expect from me? & 0 que você espera de mim? \\
\hline \multirow[t]{2}{*}{12} & $\begin{array}{l}\text { What do you think I can do } \\
\text { for you? }\end{array}$ & $\begin{array}{l}0 \text { que você acha que eu } \\
\text { posso fazer por você? }\end{array}$ & $\begin{array}{l}\text { What do you think I can do } \\
\text { for you? }\end{array}$ & $\begin{array}{l}0 \text { que você acha que eu } \\
\text { posso fazer por você? }\end{array}$ \\
\hline & Explanations (Attribution) & Explicações (Atribuição) & Explanations (Attribution) & Explicações (Atribuição) \\
\hline 13 & What do you think yourself? & $\begin{array}{l}\text { Qual a sua própria opinião a } \\
\text { respeito? }\end{array}$ & What is your opinion about it? & $\begin{array}{l}\text { Qual a sua opinião a respeito } \\
\text { dos seus sintomas? }\end{array}$ \\
\hline 14 & $\begin{array}{l}\text { Do you yourself have } \\
\text { any explanation for your } \\
\text { complaints? }\end{array}$ & $\begin{array}{c}\text { Tem alguma explicação para } \\
\text { as suas queixas? }\end{array}$ & $\begin{array}{l}\text { Do you have any explanation } \\
\text { for your complaints? }\end{array}$ & $\begin{array}{c}\text { Você tem alguma explicação } \\
\text { para os seus sintomas? }\end{array}$ \\
\hline \multirow[t]{2}{*}{15} & $\begin{array}{l}\text { Do you sometimes think "if it } \\
\text { isn't this or that"? }\end{array}$ & $\begin{array}{l}\text { Você às vezes pensa "se não } \\
\text { é isto ou aquilo"? }\end{array}$ & $\begin{array}{l}\text { Do you sometimes think "if it } \\
\text { is not this or is it that"? }\end{array}$ & $\begin{array}{c}\text { Você às vezes pensa se seus } \\
\text { sintomas não seriam isto ou } \\
\text { aquilo? }\end{array}$ \\
\hline & $\begin{array}{l}\text { Thinking about Complaints } \\
\text { / Thinking That Worsens } \\
\text { Complaints (Catastrophizing) }\end{array}$ & $\begin{array}{l}\text { Pensando sobre as queixas } \\
\text { / pensando o que piora as } \\
\text { queixas (Catastrofização) }\end{array}$ & $\begin{array}{l}\text { Thinking about the } \\
\text { complaints / thinking } \\
\text { about what worsens the } \\
\text { complaints (catastrophizing) }\end{array}$ & $\begin{array}{l}\text { Pensando sobre as queixas } \\
\text { / pensando o que piora as } \\
\text { queixas (Catastrofização) }\end{array}$ \\
\hline 16 & $\begin{array}{l}\text { How do you feel when you } \\
\text { have symptoms? }\end{array}$ & $\begin{array}{c}\text { Como você se sente quando } \\
\text { tem os sintomas? }\end{array}$ & $\begin{array}{l}\text { How do you feel when you } \\
\text { have the symptoms? }\end{array}$ & $\begin{array}{c}\text { Como você se sente quando } \\
\text { tem os sintomas? }\end{array}$ \\
\hline 17 & $\begin{array}{l}\text { What do you think at that } \\
\text { moment? }\end{array}$ & $\begin{array}{c}0 \text { que você pensa nesses } \\
\text { momentos? }\end{array}$ & $\begin{array}{l}\text { What do you think at these } \\
\text { moments? }\end{array}$ & $\begin{array}{c}0 \text { que você pensa nos } \\
\text { momentos que tem os } \\
\text { sintomas? }\end{array}$ \\
\hline \multirow[t]{2}{*}{18} & How do you react? & Como você reage? & How do you react? & Como você reage? \\
\hline & $\begin{array}{l}\text { Ideas about Personal } \\
\text { Influence on Complaints } \\
\text { (Self-Efficacy) }\end{array}$ & $\begin{array}{c}\text { Ideias sobre influência } \\
\text { pessoal sobre as queixas } \\
\text { (autoeficácia) }\end{array}$ & $\begin{array}{l}\text { Ideas about personal } \\
\text { influence on complaints } \\
\text { (self-efficacy) }\end{array}$ & $\begin{array}{l}\text { Ideias sobre influência } \\
\text { pessoal nas queixas } \\
\text { (autoeficácia) }\end{array}$ \\
\hline 19 & $\begin{array}{l}\text { Do you personally have any } \\
\text { influence on the complaint? }\end{array}$ & $\begin{array}{c}\text { Você pessoalmente tem } \\
\text { alguma influência sobre suas } \\
\text { queixas? }\end{array}$ & $\begin{array}{l}\text { Do you personally have any } \\
\text { influence on your complaints? }\end{array}$ & $\begin{array}{c}\text { Você acha que tem alguma } \\
\text { influência sobre seus } \\
\text { sintomas? }\end{array}$ \\
\hline 20 & $\begin{array}{c}\text { Can you positively influence } \\
\text { the complaint? }\end{array}$ & $\begin{array}{c}\text { Você é capaz influenciar } \\
\text { positivamente as suas } \\
\text { queixas? }\end{array}$ & $\begin{array}{l}\text { Are you able to positively } \\
\text { influence in your complaints? }\end{array}$ & $\begin{array}{c}\text { Você acha que é capaz } \\
\text { influenciar positivamente os } \\
\text { seus sintomas? }\end{array}$ \\
\hline
\end{tabular}

(To be continued) 
Table 1 - The transcultural adaptation of the SCEBS method

\begin{tabular}{|c|c|c|c|c|}
\hline $\mathrm{N}^{\circ}$ & Original & T12 & RT12 & Final Vesion \\
\hline 21 & If so, how? & Se é, como? & If so, how? & Se sim, de que maneira? \\
\hline 22 & $\begin{array}{l}\text { Is there anything you yourself } \\
\text { can do to reduce your } \\
\text { complaint? }\end{array}$ & $\begin{array}{l}\text { Existe alguma coisa que } \\
\text { você mesmo pode fazer para } \\
\text { reduzir as suas queixas? }\end{array}$ & $\begin{array}{l}\text { Is there anything you can do } \\
\text { to reduce your complaints? }\end{array}$ & $\begin{array}{c}\text { Existe alguma coisa que você } \\
\text { mesmo possa fazer para } \\
\text { reduzir os seus sintomas? }\end{array}$ \\
\hline 23 & $\begin{array}{l}\text { Do complaints resolve more } \\
\text { quickly when you rest? }\end{array}$ & $\begin{array}{l}\text { Os problemas são resolvidos } \\
\text { mais rapidamente quando } \\
\text { você fica de repouso? }\end{array}$ & $\begin{array}{l}\text { Are your complaints solved } \\
\text { quicker when you rest? }\end{array}$ & $\begin{array}{c}\text { Os seus sintomas melhoram } \\
\text { mais rapidamente quando } \\
\text { você fica de repouso? }\end{array}$ \\
\hline 24 & $\begin{array}{l}\text { Do complaints lessen when } \\
\text { you think about something or } \\
\text { someone else? }\end{array}$ & $\begin{array}{l}\text { As suas queixas diminuem } \\
\text { quando você pensa em } \\
\text { alguma coisa ou alguém? }\end{array}$ & $\begin{array}{c}\text { Do your complaints get better } \\
\text { when you think of something } \\
\text { or someone? }\end{array}$ & $\begin{array}{l}\text { Os seus sintomas diminuem } \\
\text { quando você pensa em } \\
\text { alguma coisa ou em alguém? }\end{array}$ \\
\hline A.2.2. & $\mathbf{E}=$ Emotion & 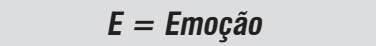 & $\mathbf{E}=$ Emotion & 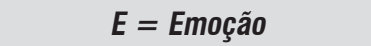 \\
\hline 25 & $\begin{array}{l}\text { Given that you have these } \\
\text { complaints, how do you feel } \\
\text { about it? }\end{array}$ & $\begin{array}{c}\text { Visto que você tem essas } \\
\text { queixas, como você se } \\
\text { sente? }\end{array}$ & $\begin{array}{c}\text { Given that you have these } \\
\text { complaints, how do you feel } \\
\text { about it? }\end{array}$ & $\begin{array}{l}\text { Visto que você tem esses } \\
\text { sintomas, como você se } \\
\text { sente em relação a eles? }\end{array}$ \\
\hline 26 & $\begin{array}{l}\text { Do the complaints disturb } \\
\text { your emotional balance? }\end{array}$ & $\begin{array}{l}\text { As queixas perturbam seu } \\
\text { equilibrio emocional? }\end{array}$ & $\begin{array}{l}\text { Do the complaints disturb } \\
\text { your emotional balance? }\end{array}$ & $\begin{array}{c}\text { Você acha que seus sintomas } \\
\text { afetam seu equilibrio } \\
\text { emocional? }\end{array}$ \\
\hline 27 & Are you insecure? & Você está inseguro(a)? & Are you insecure? & Você se sente inseguro(a)? \\
\hline 28 & Are you depressed? & Você está deprimido(a)? & Are you depressed? & $\begin{array}{l}\text { Você se considera } \\
\text { deprimido(a)? }\end{array}$ \\
\hline 29 & Are you anxious? & Você está ansioso(a)? & Are you anxious? & $\begin{array}{l}\text { Você se considera } \\
\text { ansioso(a)? }\end{array}$ \\
\hline 30 & $\begin{array}{c}\text { Do you ever feel overwhelmed } \\
\text { by the complaints? }\end{array}$ & $\begin{array}{c}\text { Você já se sentiu } \\
\text { sobrecarregado pelas suas } \\
\text { queixas? }\end{array}$ & $\begin{array}{l}\text { Have you ever felt } \\
\text { overwhelmed by your } \\
\text { complaints? }\end{array}$ & $\begin{array}{l}\text { Você já se sentiu } \\
\text { sobrecarregado pelos seus } \\
\text { sintomas? }\end{array}$ \\
\hline A.2.3. & B = Behavior & $B=$ Comportamento & B = Behavior & $B=$ Comportamento \\
\hline & Dealing with the Complaint & Lidando com a queixa & Dealing with the complaint & Lidando com a queixa \\
\hline 31 & $\begin{array}{l}\text { What do you do if you have } \\
\text { symptoms? }\end{array}$ & $\begin{array}{c}0 \text { que você faz se tem } \\
\text { sintomas? }\end{array}$ & $\begin{array}{l}\text { What do you do if you have } \\
\text { symptoms? }\end{array}$ & $\begin{array}{c}\text { O que você faz quando tem } \\
\text { os sintomas? }\end{array}$ \\
\hline 32 & $\begin{array}{l}\text { What do you do to reduce } \\
\text { symptoms? }\end{array}$ & $\begin{array}{c}\text { O que você faz para reduzir } \\
\text { os sintomas? }\end{array}$ & $\begin{array}{l}\text { What do you do to reduce the } \\
\text { symptoms? }\end{array}$ & $\begin{array}{c}\text { O que você faz para reduzir } \\
\text { os sintomas? }\end{array}$ \\
\hline \multirow[t]{2}{*}{33} & $\begin{array}{l}\text { To what extent is this } \\
\text { successful? }\end{array}$ & Até que ponto isso funciona? & To what extent does it work? & Até que ponto isso funciona? \\
\hline & Limitations to Activities & Limitações de atividades & Activities limitations & Limitações de atividades \\
\hline 34 & $\begin{array}{l}\text { Which activities are hindered } \\
\text { by your complaints? }\end{array}$ & $\begin{array}{c}\text { Quais atividades são } \\
\text { prejudicadas pela suas } \\
\text { queixas? }\end{array}$ & $\begin{array}{l}\text { Which activities are hindered } \\
\text { by your complaints? }\end{array}$ & $\begin{array}{l}\text { Quais atividades são } \\
\text { prejudicadas pelos seus } \\
\text { sintomas? }\end{array}$ \\
\hline \multirow[t]{2}{*}{35} & To what extent? & Até que ponto? & To what extent? & $\begin{array}{l}\text { Até que ponto os sintomas } \\
\text { prejudicam suas atividades? }\end{array}$ \\
\hline & Avoidance & Evitação & Avoidance & Evitação \\
\hline 36 & $\begin{array}{l}\text { What do not you do or no } \\
\text { longer do when you have } \\
\text { symptoms? }\end{array}$ & $\begin{array}{c}\text { O que você não faz ou deixou } \\
\text { de fazer quando tem os } \\
\text { sintomas? }\end{array}$ & $\begin{array}{l}\text { What do not you do or no } \\
\text { longer do when you have } \\
\text { symptoms? }\end{array}$ & $\begin{array}{c}\text { O que você não faz ou deixa } \\
\text { de fazer quando tem os } \\
\text { sintomas? }\end{array}$ \\
\hline 37 & Since when? & Desde quando? & Since when? & Desde quando? \\
\hline 38 & $\begin{array}{l}\text { Are you anxious about } \\
\text { particular activities? }\end{array}$ & $\begin{array}{l}\text { Você fica ansioso sobre } \\
\text { alguma atividade específica? }\end{array}$ & $\begin{array}{l}\text { Do you get anxious about } \\
\text { some specific activity? }\end{array}$ & $\begin{array}{l}\text { Você fica ansioso em } \\
\text { relação a alguma atividade } \\
\text { específica? }\end{array}$ \\
\hline 39 & $\begin{array}{l}\text { What do other people notice } \\
\text { about your behavior when } \\
\text { you have symptoms? }\end{array}$ & $\begin{array}{c}0 \text { que as outras } \\
\text { pessoas notam em seu } \\
\text { comportamento quando você } \\
\text { tem os sintomas? }\end{array}$ & $\begin{array}{l}\text { What other people notice } \\
\text { about your behavior when } \\
\text { you have the symptoms? }\end{array}$ & $\begin{array}{c}\text { o que as outras pessoas } \\
\text { percebem em seu } \\
\text { comportamento quando você } \\
\text { tem os sintomas? }\end{array}$ \\
\hline
\end{tabular}

(To be continued) 
Table 1 - The transcultural adaptation of the SCEBS method

(Conclusion)

\begin{tabular}{|c|c|c|c|c|}
\hline $\mathrm{N}^{\circ}$ & Original & T12 & RT12 & Final Vesion \\
\hline & Talking about Complaints & $\begin{array}{c}\text { Conversando sobre os } \\
\text { problemas }\end{array}$ & Talking about complaints & Falando sobre as queixas \\
\hline 40 & $\begin{array}{c}\text { Do you talk about your } \\
\text { complaints? With whom? } \\
\text { How often? }\end{array}$ & $\begin{array}{l}\text { Você conversa sobre suas } \\
\text { queixas? Com quem? Com } \\
\text { que frequência? }\end{array}$ & $\begin{array}{l}\text { Do you talk about your } \\
\text { complaints? With whom? } \\
\text { How often? }\end{array}$ & $\begin{array}{c}\text { Você conversa sobre seus } \\
\text { sintomas? Com quem? Com } \\
\text { que frequência? }\end{array}$ \\
\hline 41 & What do you tell them? & O que você diz a eles? & What do you tell them? & 0 que você diz a ele(a)(s)? \\
\hline A.3. & $S=$ Social Dimension & $S=$ Dimensão Social & $S=$ Social Dimension & $S=$ Dimensão Social \\
\hline 42 & $\begin{array}{l}\text { Do the people around you } \\
\text { notice when you have } \\
\text { complaints? }\end{array}$ & $\begin{array}{c}\text { As pessoas ao seu redor } \\
\text { notam quando você tem } \\
\text { queixas? }\end{array}$ & $\begin{array}{l}\text { Do the people around you } \\
\text { notice when you have } \\
\text { complaints? }\end{array}$ & $\begin{array}{l}\text { As pessoas ao seu redor } \\
\text { percebem quando você tem } \\
\text { os sintomas? }\end{array}$ \\
\hline 43 & What do they notice? & 0 que elas notam? & What do they notice? & 0 que elas percebem? \\
\hline 44 & $\begin{array}{l}\text { How do you react to your } \\
\text { complaints? }\end{array}$ & $\begin{array}{c}\text { Como você reage a suas } \\
\text { queixas? }\end{array}$ & $\begin{array}{l}\text { How do you react to your } \\
\text { complaints? }\end{array}$ & $\begin{array}{c}\text { Como você reage aos seus } \\
\text { sintomas? }\end{array}$ \\
\hline 45 & $\begin{array}{l}\text { What do the people around } \\
\text { you think about your } \\
\text { complaints? }\end{array}$ & $\begin{array}{c}0 \text { que as pessoas ao seu } \\
\text { redor pensam sobre as suas } \\
\text { queixas? }\end{array}$ & $\begin{array}{l}\text { What do the people around } \\
\text { you think about your } \\
\text { complaints? }\end{array}$ & $\begin{array}{l}\text { 0 que as pessoas ao seu } \\
\text { redor pensam sobre os seus } \\
\text { sintomas? }\end{array}$ \\
\hline 46 & $\begin{array}{l}\text { How do the people around } \\
\text { you react to your complaints? }\end{array}$ & $\begin{array}{c}\text { Como as pessoas ao seu } \\
\text { redor reagem as suas } \\
\text { queixas? }\end{array}$ & $\begin{array}{l}\text { How do the people around } \\
\text { you react about your } \\
\text { complaints? }\end{array}$ & $\begin{array}{c}\text { Como as pessoas ao seu } \\
\text { redor reagem aos seus } \\
\text { sintomas? }\end{array}$ \\
\hline 47 & $\begin{array}{l}\text { Where does your partner } \\
\text { think that your complaints } \\
\text { come from? }\end{array}$ & $\begin{array}{l}\text { Seu parceiro tem ideia do } \\
\text { motivo das suas queixas? }\end{array}$ & $\begin{array}{l}\text { Where does your partner } \\
\text { think that your complaints } \\
\text { come from? }\end{array}$ & $\begin{array}{l}\text { Para o seu parceiro, de } \\
\text { onde é que surgem os seus } \\
\text { sintomas? }\end{array}$ \\
\hline 48 & $\begin{array}{l}\text { How did the people around } \\
\text { you react when you told them } \\
\text { what the doctor said? }\end{array}$ & $\begin{array}{c}\text { Como as pessoas ao seu } \\
\text { redor reagiram quando você } \\
\text { Ihes contou o que o médico } \\
\text { disse? }\end{array}$ & $\begin{array}{l}\text { How people around you react } \\
\text { when you told them what the } \\
\text { doctor said? }\end{array}$ & $\begin{array}{c}\text { Como as pessoas ao seu } \\
\text { redor reagiram quando você } \\
\text { Ihes contou o que o médico } \\
\text { disse? }\end{array}$ \\
\hline 49 & $\begin{array}{l}\text { How do you now feel about } \\
\text { this? }\end{array}$ & $\begin{array}{c}\text { Como você se sente sobre } \\
\text { isso agora? }\end{array}$ & $\begin{array}{l}\text { How do you feel about it } \\
\text { now? }\end{array}$ & $\begin{array}{l}\text { Como você se sente sobre } \\
\text { isso agora? }\end{array}$ \\
\hline 50 & $\begin{array}{l}\text { Do the complaints affect your } \\
\text { social life? }\end{array}$ & $\begin{array}{c}\text { As queixas afetam sua vida } \\
\text { social? }\end{array}$ & $\begin{array}{l}\text { Do the complaints affect your } \\
\text { social life? }\end{array}$ & $\begin{array}{c}\text { Os sintomas afetam sua vida } \\
\text { social? }\end{array}$ \\
\hline 51 & $\begin{array}{l}\text { Did you need to adapt your } \\
\text { work/hobby/ sport to your } \\
\text { complaints? }\end{array}$ & $\begin{array}{c}\text { Você precisou adaptar seu } \\
\text { trabalho /lazer/esporte a sua } \\
\text { queixa? }\end{array}$ & $\begin{array}{l}\text { Did you have to adapt your } \\
\text { work / leisure / sport to your } \\
\text { complaint? }\end{array}$ & $\begin{array}{l}\text { Você precisou adaptar seu } \\
\text { trabalho /lazer/esporte aos } \\
\text { seus sintomas? }\end{array}$ \\
\hline
\end{tabular}

Pre-test

The SCEBS method was applied to 50 healthy individuals, of which 41 were female (82\%). The mean age was 26.1 years (range $=19$ to $51, \mathrm{SD}=6.9$ ). The degree of education in this group was characterized by: 38 (76\%) incomplete graduate level, nine (18\%) complete graduate level, two (4\%) master's degree, and one $(2 \%) \mathrm{PhD}$ level. The symptomatic group consisted of 50 participants, 43 females (86\%). The mean age was 57.7 years (range $=29$ to $82, \mathrm{SD}=13.3$ ). Musculoskeletal pain was distributed as follows: $30 \%$ had lower back pain, $6 \%$ neck pain, and $64 \%$ lower back and neck pain. In relation to schooling, the group of patients was composed of 21 (42\%) with incomplete elementary school, five (10\%) with first grade complete, one (2\%) with incomplete high school, 17 (34\%), with a high school diploma (34\%), and six $(12 \%)$ with a graduate level education.

In the pre-test, the pre-final version of the instrument was tested to evaluate semantic, idiomatic, experimental, and conceptual equivalences. All items were satisfactorily understood, both by the control group and by the symptomatic group, with the items all presenting scores above 4 points. In the healthy group only question Q15 (mean $=4.38, \mathrm{SD}=0.98$ ) had a mean value lower than 4.5 points. In the symptomatic group, Q21 (mean $=4.34, \mathrm{SD}=1.33$ ), $\mathrm{Q} 21$ (mean $=4.24, \mathrm{SD}=1.34)$, and Q24 $($ mean $=4.42$, $\mathrm{SD}=1.31$ ) obtained mean values lower than 4.5 points. The final version of the instrument is presented in Figure 1. Figure 2 shows the questionnaire in English. 
Versão Brasileira do método SCEBS
A.1. $S$ = DIMENSÃO SOMÁTICA
(1) Quais são as suas queixas?
(2) Quando os seus sintomas começaram?
(3) Quais são o tipo, a localização e a intensidade dos seus sintomas?
(4) Com que frequência os sintomas ocorrem?
(5) Quanto tempo duram esses sintomas?
(6) Você já teve esses sintomas antes?
(7) Você consegue movimentar suas costas ou pescoço?
(8) Você teve alguma dificuldade para movimentar?
(9) 0 que mostram os resultados do raio-X?
(10) 0 que mostram os testes de laboratório?

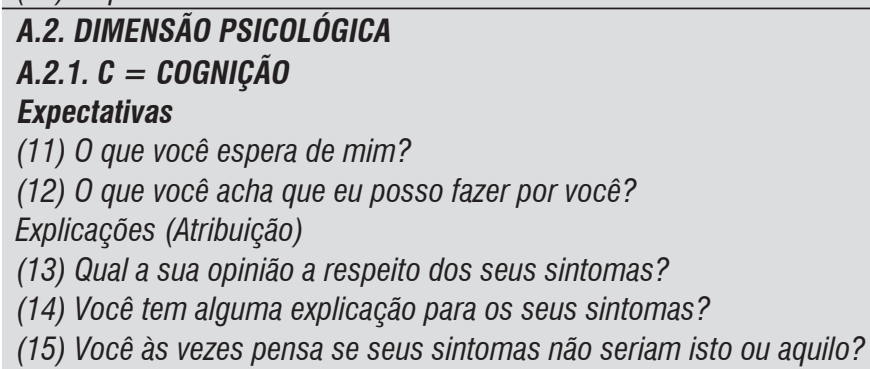

Pensando sobre as queixas / pensando o que piora as queixas (Catastrofização)

(16) Como você se sente quando tem os sintomas?

(17) 0 que você pensa nos momentos que tem os sintomas?

(18) Como você reage?

Ideias sobre influência pessoal nas queixas (autoeficácia)

(19) Você acha que tem alguma influência sobre seus sintomas?

(20) Você acha que é capaz influenciar positivamente os seus sintomas?

(21) Se sim, de que maneira?

(22) Existe alguma coisa que você mesmo possa fazer para reduzir os seus sintomas?

(23) Os seus sintomas melhoram mais rapidamente quando você fica de repouso?

(24) Os seus sintomas diminuem quando você pensa em alguma coisa ou em alguém?
A.2.2. $E=$ EMOÇÃO
(25) Visto que você tem esses sintomas, como você se sente em relação a eles?
(26) Você acha que seus sintomas afetam seu equilibrio emocional?
(27) Você se sente inseguro(a)?
(28) Você se considera deprimido(a)?
(29) Você se considera ansioso(a)?
(30) Você já se sentiu sobrecarregado pelos seus sintomas?

\section{A.2.3. B = COMPORTAMENTO \\ Lidando com os sintomas \\ (31) 0 que você faz quando tem os sintomas? \\ (32) 0 que você faz para reduzir os sintomas? \\ (33) Até que ponto isso funciona? \\ Limitações de atividades}

(34) Quais atividades são prejudicadas pelos seus sintomas?

(35) Até que ponto os sintomas prejudicam suas atividades?

\section{Evitação}

(36) 0 que você não faz ou deixa de fazer quando tem os sintomas?

(37) Desde quando?

(38) Você fica ansioso em relação a alguma atividade específica?

(39) 0 que as outras pessoas percebem em seu comportamento quando você tem os sintomas?

Falando sobre as queixas

(40) Você conversa sobre seus sintomas? Com quem? Com que frequência?

(41) 0 que você diz a ele(a)(s)? 


\section{Versão Brasileira do método SCEBS}

\section{A.3. $S$ = DIMENSÃO SOCIAL}

(42) As pessoas ao seu redor percebem quando você tem os sintomas?

(43) 0 que elas percebem?

(44) Como você reage aos seus sintomas?

(45) 0 que as pessoas ao seu redor pensam sobre os seus sintomas?

(46) Como as pessoas ao seu redor reagem aos seus sintomas?

(47) Para o seu parceiro, de onde é que surgem os seus sintomas?

(48) Como as pessoas ao seu redor reagiram quando você Ihes contou o que o médico disse?

(49) Como você se sente sobre isso agora?

(50) Os sintomas afetam sua vida social?

(51) Você precisou adaptar seu trabalho /lazer/esporte aos seus sintomas?

Figure 1 - Portuguese version of the SCEBS method.

\section{A. SCEBS Method}

\section{A.1. $S$ = Somatic Dimension}

(1) What are your complaints?

(2) When did the complaints begin?

(3) What are the nature, the location, and the intensity of the complaints?

(4) How often do the symptoms occur?

(5) How long do the symptoms last?

(6) Have you had these symptoms before?

(7) Can you move your back/neck

(8) Have you experienced any stiffness?

(9) What do the X-ray results show?

(10) What do laboratory tests show?

\section{A.2. Psychological Dimension}

\section{A.2.1. $C=$ Cognition}

\section{Expectations}

(11) What do you expect from me?

(12) What do you think I can do for you?

Explanations (Attribution)

(13) What do you think yourself?

(14) Do you yourself have any explanation for your complaints?

(15) Do you sometimes think "if it isn't this or that"?

Thinking about Complaints/Thinking That Worsens Complaints (Catastrophizing)

(16) How do you feel when you have symptoms?

(17) What do you think at that moment?

(18) How do you react?

Ideas about Personal Influence on Complaints (Self-Efficacy)

(19) Do you personally have any influence on the complaint?

(20) Can you positively influence the complaint?

(21) If so, how?

(22) Is there anything you yourself can do to reduce your complaint?

(23) Do complaints resolve more quickly when you rest?

(24) Do complaints lessen when you think about something or someone else?

\section{A.2.2. $E$ = Emotion}

(25) Given that you have these complaints, how do you feel about it?

(26) Do the complaints disturb your emotional balance?

(27) Are you insecure?

(28) Are you depressed?

(29) Are you anxious?

(30) Do you ever feel overwhelmed by the complaints? 


\section{A. SCEBS Method}

A.2.3. B = Behavior
Dealing with the Complaint
(31) What do you do if you have symptoms?
(32) What do you do to reduce symptoms?
(33) To what extent is this successful?
Limitations to Activities
(34) Which activities are hindered by your complaints?
(35) To what extent?
Avoidance
(36) What do not you do or no longer do when you have symptoms?
(37) Since when?
(38) Are you anxious about particular activities?
(39) What do other people notice about your behavior when you have symptoms?
Talking about Complaints
(40) Do you talk about your complaints? With whom? How often?
(41) What do you tell them?
A.3. S = Social Dimension
(42) Do the people around you notice when you have complaints?
(43) What do they notice?
(44) How do you react to your complaints?
(45) What do the people around you think about your complaints?
(46) How do the people around you react to your complaints?
(47) Where does your partner think that your complaints come from?
(48) How did the people around you react when you told them what the doctor said?
(49) How do you now feel about this?
(50) Do the complaints affect your social life?
(51) Did you need to adapt your work/hobby/sport to your complaints?

Figure 2 - SCEBS method.

\section{Discussion}

The semantic translation and validation of the SCEBS method proved to be adequate even though there were differences in schooling and mean age among the groups included in this study. The comprehension level obtained using the transcultural adaptation process was considered satisfactory for application of the final version in clinical practice. We included a sample young and undergraduate students in the healthy group to consider as a gold standard in the understanding of the items. We emphasize that the availability of this method can help to guide health professionals in the use of the BPS model when interviewing patients with lumbar or neck pain. It should be noted that this method is of clinical importance because the BPS model considers the individual to be a complex system, the health of which is subject to multi-factor interactions [18].

To date, the SCEBS method is the first instrument to be submitted to the process of cross-cultural adaptation into Brazilian Portuguese that guides the professional to the assessment of the patient based on the BPS model. The implementation of the SCEBS method in clinical practice may facilitate the patientprofessional relationship by scaffolding discussion of different factors that contributes to illness, establishing concrete information, and constructing a safe and open relationship [19].

We emphasize the importance of such an expanded perspective in the evaluation of people with pain because the BPS factors all contribute to the transition and persistence of pain and central sensitization [20 - 22]. Neurophysiological changes in central pain processing may be responsible for or influenced by behavioral, emotional, social, and cognitive changes [23]. For example, catastrophizing [24], pain-related anxiety [25], depressive feelings and stress [26], and decreased self-efficacy [23] are known to contribute to pain and restriction in daily activities [27]. In this way, the initial examination should take into account both the biological factors that can contribute to bottom-up stimuli as well as the psychosocial 
ones that contribute with facilitating mechanisms (top-down). Therefore, a complete clinical BPS assessment is necessary to understand the pain experience and allow an individualized and patientcentered approach.

However, evaluation based on the BPS model and identification of the multiple factors that influence pain can be difficult, with some factors being valued more than others. Oostendorp et al. [12] observed that experienced physiotherapists who believed that they were applying the BPS model in their assessments did not address several aspects of the psychosocial dimensions. During the evaluation, $98.1 \%$ of the professionals investigated information regarding the somatic dimension. However, regarding the psychological and social dimension, the approach was inadequate, with $42.5 \%$ of professionals addressing aspects related to cognition, $37.9 \%$ behavior, $26.8 \%$ emotions, and $17.6 \%$ social aspects.

Despite recognition of the need to implement the BPS model in clinical practice, there is still a great distance between theory and practice [27 - 30]. In a study conducted in Australia with physiotherapists experienced in the management of people with musculoskeletal disorders, participants emphasized that limited knowledge about what psychosocial factors mean, limited training within this model, lack of formal training, and the need of additional specific tools were the main difficulties in adopting the BPS model. The SCEBS method may encourage clinicians to adopt a broader evaluation model, as well as being a tool of easy applicability and comprehension $[12,21]$.

Wijma et al. [26] describe in a practical way the use of the SCEBS method in BPS evaluation of patients with persistent pain, and emphasize that the instrument is able to define which BPS factors are contributing to the chronicity of pain and to direct the most effective treatment plan. The recommendation of the Dutch College of General Practitioners (NHG) is that the dimensions of the SCEBS method should be used as parameters for monitoring individuals with persistent pain [19].

The main limitations of this study involved the impossibility of more complex analysis in addition to semantic validation, such as the psychometric evaluation of the instrument, and the impossibility of comparing this study with validated translations into other languages as there are only two considered original, one in Dutch and one in English [12]. The
SCEBS method was applied only to people with lower back pain and neck pain, and it is necessary to further adapt it to other populations with other painful conditions, especially those of nonmusculoskeletal origin.

\section{Conclusion}

The process of cross-cultural adaptation of the SCEBS method gave rise to an instrument that proved to be easy to understand. The final version obtained adequate semantic validation, independent of schooling or age variables, and can be considered adequate for clinical use.

\section{References}

1. Merskey $\mathrm{H}$, Bogduk $\mathrm{N}$, editors. Classification of chronic pain: descriptions of chronic pain syndromes and definitions of pain terms. 2 nd ed. Seattle: IASP PRESS; 2002.

2. Williams AC, Craig KD. Updating the definition of pain. Pain. 2016;157(11):2420-3.

3. Belloch A, Olabarría B. El modelo bio-psico-social: Un marco de referencia necesario para el psicólogo clínico. Clín Salud. 1993;4(2):181-90.

4. Ramond-Roquin $\mathrm{A}$, Bouton $\mathrm{C}$, Bègue $\mathrm{C}$, Petit $\mathrm{A}$, Roquelaure Y, Huez JF. Psychosocial Risk Factors, Interventions, and Comorbidity in Patients with Non-Specific Low Back Pain in Primary Care: Need for Comprehensive and Patient-Centered Care. Front Med (Lausanne). 2015;2:73.

5. MarquezJO. A dor e os seus aspectos multidimensionais. Cienc Cult. 2011;63(2):28-32.

6. Alcântara MA, Sampaio RF, Souza MAP, Silva FCM, Kirkwood RN. Chronic pain profile: An interaction between biological and psychosocial factors. Pain Stud Treat. 2013;1(2):9-16.

7. Schmitz A. Eu vejo a mente como vejo o corpo? A percepção da equipe de saúde de um hospital geral acerca das influências dos aspectos psicológicos no processo de adoecimento de pacientes hospitalizados [undergraduate thesis]. Palhoça: Universidade do Sul de Santa Catarina; 2009. 
8. Cruz CSS, Fernandes DRF, Pimenta MLP, Calegário LO. Do pensamento clínico, segundo Foucault, ao resgate do modelo biopsicossocial: uma análise reflexiva. Rev Univ Vale Rio Verde. 2013;11(1):30-9.

9. Sardá Jr JJ, Nicholas MK, Pimenta CAM, Asghari A. Preditores biopsicossociais de dor, incapacidade e depressão em pacientes brasileiros com dor crônica. Rev Dor. 2012;13(2):111-8.

10. Hadjistavropoulos T, Craig KD, Duck S, Cano A, Goubert L, Jackson PL, et al. Biopsychosocial Formulation of Pain Communication. Psychol Bull. 2011;137(6):910-39.

11. Marco MA. Do modelo biomédico ao modelo biopsicossocial: um projeto de educação permanente. Rev Bras Educ Med. 2006;30(1):60-72.

12. Oostendorp RAB, Elvers H, Mikołajewska E, Laekeman M, Trijffel EV, Samwel H, et al. Manual Physical Therapists' Use of Biopsychosocial History Taking in the Management of Patients with Back or Neck Pain in Clinical Practice. Sci World J. 2015;2015:170463.

13. Guillemin F, Bombardier C, Beaton D. Cross-cultural adaptation of health-related quality of life measures: literature review and proposed guidelines. J Clin Epidemiol. 1993;46(12):1417-32.

14. Beaton DE, Bombardier C, Guillemin F, Ferraz MB. Guidelines for the process of cross-cultural adaptation of self-report measures. Spine (Phila Pa 1976). 2000;25(24):3186-91.

15. Wild D, Grove A, Martin M, Eremenco S, McElroy S, Verjee-Lorenz A, et al. Principles of Good Practice for the Translation and Cultural Adaptation Process for Patient-Reported Outcomes (PRO) Measures: report of the ISPOR Task Force for Translation and Cultural Adaptation. Value Health. 2005;8(2):94-104.

16. Nusbaum L, Natour J, Ferraz MB, Goldenberg J. Translation, adaptation and validation of the RolandMorris questionnaire-Brazil Roland-Morris. Braz J Med Biol Res. 2001;34(2):203-10.

17. Grassi-Oliveira R, Stein LM, Pezzi JC. Tradução e validação de conteúdo da versão em português do Childhood Trauma Questionnaire. Rev Saude Publica. 2006;40(2):249-55.
18. Engel GL. The clinical application of the biopsychosocial model. J Med Philos. 1981;6(2):101-23.

19. Hartman TO, Blankenstein N, Molenaar B, van den Berg DB, van der Horst H, Arnold I, et al. NHG Guideline on Medically Unexplained Symptoms (MUS). Huisarts Wet. 2013;56(5):222-30.

20. Singla $\mathrm{M}$, Jones $\mathrm{M}$, Edwards I, Kumar S. Physiotherapists' assessment of patients' psychosocial status: Are we standing on thin ice? A qualitative descriptive study. Man Ther. 2015;20(2):328-34.

21. Lee H, Hübscher M, Moseley GL, Kamper SJ, Traeger AC, Mansell G, et al. How does pain lead to disability? A systematic review and meta-analysis of mediation studies in people with back and neck pain. Pain. 2015;156(6):988-97.

22. Sobral MLP, Badessa MPSG, Sobral MLP, Oliveira Jr JB. Estudo da prevalência de algias na coluna vertebral em residentes de cirurgia cardiovascular: estudo inicial. Rev Bras Med Trab. 2013;11(2):82-9.

23. Turk DC, Okifuji A. Psychological factors in chronic pain: evolution and revolution. J Consult Clin Psychol. 2002;70(3):678-90.

24. Gracely RH, Geisser ME, Giesecke T, Grant MA, Petzke F, Williams DA, et al. Pain catastrophizing and neural responses to pain among persons with fibromyalgia. Brain. 2004;127(Pt 4):835-43.

25. Rivat C, Becker C, Blugeot A, Zeau B, Mauborgne A, Pohl M, et al. Chronic stress induces transient spinal neuroinflammation, triggering sensory hypersensitivity and long-lasting anxiety-induced hyperalgesia. Pain. 2010;150(2):358-68.

26. Wijma AJ, van Wilgen CP, Meeus M, Nijs J. Clinical biopsychosocial physiotherapy assessment of patients with chronic pain: The first step in pain neuroscience education. Physiother Theory Pract. 2016;32(5):368-84.

27. Foster NE, Delitto A. Embedding psychosocial perspectives within clinical management of low back pain: integration of psychosocially informed management principles into physical therapist practice-challenges and opportunities. Phys Ther. 2011;91(5):790-803. 
28. Bishop A, Foster NE. Do physical therapists in the United Kingdom recognize psychosocial factors in patients with acute low back pain? Spine (Phila Pa 1976). 2005;30(11):1316-22.

29. Alexanders J, Anderson A, Henderson S. Musculoskeletal physiotherapists' use of psychological interventions: a systematic review of therapists' perceptions and practice. Physiotherapy. 2015;101(2):95-102.
30. Jull G. Biopsychosocial model of disease: 40 years on. Which way is the pendulum swinging? Br J Sports Med. 2017;51(16):1187-8.

Received on $08 / 17 / 2017$

Recebido em 17/08/2017

Approved on 10/04/2017

Aprovado em 04/10/2017 